\title{
Electrodeposition of Ni-Si Schottky Barriers
}

\author{
Michail E. Kiziroglou ${ }^{1}$ Member, IEEE, Alexander A. Zhukov ${ }^{2}$, Mamdouh Abdelsalam ${ }^{3}, \mathrm{Xiaoli}_{\mathrm{Li}}{ }^{1}$, \\ Peter A. J. de Groot ${ }^{2}$, Philip N. Bartlett ${ }^{3}$, and Cornelis H. de Groot ${ }^{1}$, Member, IEEE \\ ${ }^{1}$ School of Electronics and Computer Science, University of Southampton, Highfield, Southampton S017 1BJ, U.K. \\ ${ }^{2}$ School of Physics and Astronomy, University of Southampton, Highfield, Southampton SO17 1BJ, U.K. \\ ${ }^{3}$ School of Chemistry, University of Southampton, Highfield, Southampton S017 1BJ, U.K.
}

\begin{abstract}
Electrodeposition is being used to fabricate magnetic microstructures directly on patterned n-type Si wafers of various substrate resistivities. The Ni-Si Schottky barrier is characterized and found to be of high quality for relatively low $\mathrm{Si}$ resistivities (1-2 $\Omega \cdot c m)$, with extremely low reverse leakage. It is shown that a direct correlation exists among the electrodeposition potential, the roughness, and the coercivity of the films. A conductive seed layer or a back contact is not compulsory for electrodeposition on Si with resistivities up to $15 \Omega \cdot \mathrm{cm}$. This shows that electrodeposition of magnetic materials on Si might be a viable fabrication technique for magnetoresistance and spintronics applications.
\end{abstract}

Index Terms-Electrodeposition, Schottky barriers, silicon, spintronics.

\section{INTRODUCTION}

$\mathbf{T}$ HE future of spintronics and magnetoresistance applications depends heavily on the integration of magnetic materials with silicon. Schottky barriers (SBs) between magnetic metals and semiconductors are particulary useful for suppressing substrate leakage currents in magnetoresistance measurements. Furthermore, hot electron spin valve transistors require high quality SBs for their operation [1], [2]. The development of fabrication methods for high quality magnetic metal-Si SB junctions is therefore crucial for the implementation of integrated spintronic devices.

Electrodeposition is low-temperature, cost effective, and particularly attractive for its vital role in the fabrication of self-assembled magnetic nanostructures where line-of-sight techniques do not work [3]. Electrodeposition has been used in the past for the formation of magnetic layers on $\mathrm{Si} \mathrm{[4]} \mathrm{but} \mathrm{the}$ electrical behavior of the resulting structures has not yet been investigated.

In this paper, electrodeposition as a metallization technique for spintronic applications is proposed. Ni microstructures were grown directly on lithographically patterned Si substrates. The characterization of electrodeposited $\mathrm{Ni}-\mathrm{Si}$ contacts reveals that the resulting SB is superior to those fabricated by other metallization techniques [5]. The film's roughness is controlled by the electrodeposition conditions and a correlation between roughness and coercivity is found. It is also shown that electrodeposition on resistive $\mathrm{Si}$ is possible without the need of back contacts for resistivities up to $15 \Omega \cdot \mathrm{cm}$.

\section{EXPERIMENTAL PROCEDURE}

Electrodeposition of Ni on n-type, $\langle 100\rangle \mathrm{Si}$ with resistivities of $0.01-0.02 \Omega \cdot \mathrm{cm}, 1-2 \Omega \cdot \mathrm{cm}$, and $10-20 \Omega \cdot \mathrm{cm}$ was performed using a Ni sulphate bath [3]. An Autolab AUT72032 potentiostat three electrode system with a Pt counter electrode and a saturated calomel reference electrode (SCE) were used.

Digital Object Identifier 10.1109/TMAG.2005.854737
For the definition of microstructures a 20-nm thermal $\mathrm{SiO}_{2}$ was grown on the $\mathrm{Si}$ wafers and patterns were defined by photolithography. Subsequently, the wafers were immersed in a 20:1 buffered HF solution which etched the oxide and exposed $\mathrm{Si}$ areas of different shapes and sizes ranging from $2.5 \mu \mathrm{m}$ to $1.5 \mathrm{~mm}$. On some $\mathrm{Si}$ wafers ohmic Al back contacts were defined to allow for electrical measurements. This was done by $\mathrm{Al}$ evaporation followed by an anneal for $30 \mathrm{~min}$ at $450{ }^{\circ} \mathrm{C}$ in $\mathrm{H}_{2} / \mathrm{N}_{2}$. In all experiments the wafers were sawed in pieces, RCA cleaned, and dipped in 20:1 buffered HF prior to electrodeposition. The thickness of the Ni films was controlled by measuring the charge accumulated at the cathode electrode (Si substrate) during electrodeposition taking into account the hydrogen evolution effects.

The films were characterized using an optical microscope and a Topometrix $100 \mu, 10-\mathrm{Z}$ linearized Accurex atomic force microscopy (AFM) Explorer. Electrical measurements of the Ni-Si junctions where taken using a Hewlett-Packard 4155 A semiconductor parameter analyzer. Magnetic measurements were performed at room temperature using an Oxford Instruments 3001 vibrating sample magnetometer.

\section{RESUlTS AND DISCUSSION}

\section{A. Schottky Barrier Characterization}

Ni pads were electrodeposited on $\mathrm{Si}$ and the Ni-Si electrical contact was measured for two different $\mathrm{Si}$ resistivities. For $\mathrm{Si}$ resistivities of $0.01-0.02 \Omega \cdot \mathrm{cm}$, the SB is very weak. In contrast, for $\mathrm{Si}$ with $1-2 \Omega \cdot \mathrm{cm}$ excellent SBs are achieved with very low reverse bias current, 6-7 orders of magnitude smaller than the forward bias current. Fig. 1 illustrates typical J-V curves of $\mathrm{Ni}-\mathrm{Si}$ junctions for both cases.

For the characterization of the high resistivity $\mathrm{Ni}-\mathrm{Si} \mathrm{SB}$, the saturation current density $J_{S}$ was derived by extrapolation of the linear forward part to the $y$-axis and it was found to be $1 \mu \mathrm{A} \cdot \mathrm{cm}^{-2}$, in agreement with the reverse bias current. The SB height is $0.71 \mathrm{~V}$ and the ideality factor $(\eta)$ is around 1.1 at low voltages. 


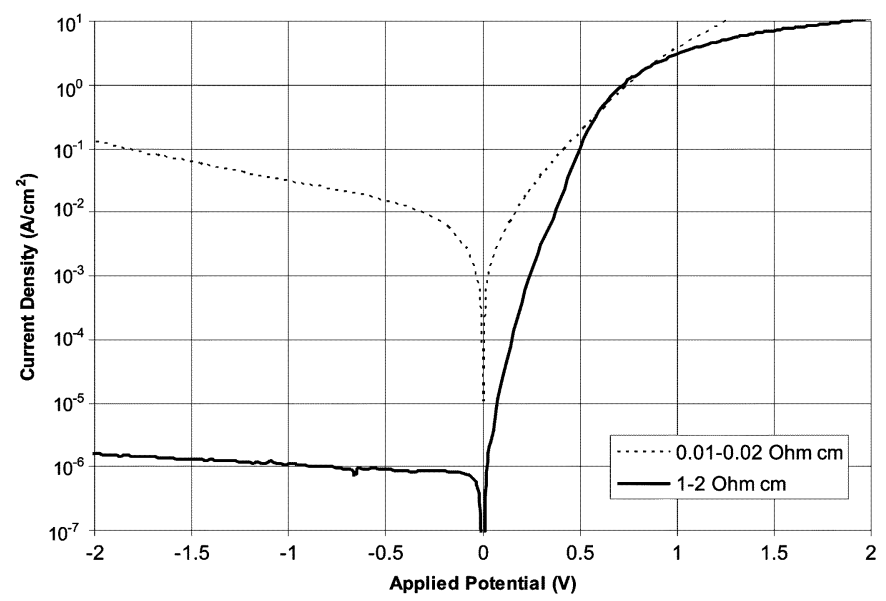

Fig. 1. J-V curves of electrodeposited Ni-Si contacts showing excellent SB for Si resistivity $1-2 \Omega \cdot \mathrm{cm}$. The contacts are circular with diameter $0.04 \mathrm{~cm}$.

Some of the junctions exhibited a deviation from linearity appearing in the vicinity of $0.3 \mathrm{~V}$ at forward bias. This deviation can be explained phenomenologically by considering an inhomogeneous junction. Such junctions consist of several SBs in parallel. These SBs can be taken into account by assuming that the total current is the resultant of two currents running in parallel

$$
I_{\text {total }}=I_{1}+I_{2}
$$

The first current, $I_{1}$, corresponds to an ideal SB current and can be expressed as

$$
I_{1}=I_{S 1} \cdot e^{\left(V-I_{1} \cdot R_{S 1}\right) \cdot \frac{q}{k \cdot T}}
$$

The second current, $I_{2}$, contains nonideal effects of a practical Schottky diode

$$
I_{2}=I_{S 2} \cdot e^{\left(V-I_{2} \cdot R_{S 2}\right) \cdot \frac{q}{\eta \cdot k \cdot T}} .
$$

In the above equations, $R_{S 1}$ and $R_{S 2}$ are the series resistances, $I_{S 1}$ and $I_{S 2}$ the saturation currents, $q$ the elementary charge, $k$ the Boltzmann constant, and $T$ the absolute temperature. The variation of the ideality factor $\eta$ in (3) gives $I-V$ curves with different forward bias deviations. In such a manner, the $I-V$ characteristics of nominal identical Schottky contacts prepared on a single substrate can be fitted as shown in Fig. 2.

The sum of the $I_{S 1}$ and $I_{S 2}$ values used in the model corresponds to a current of $4 \mathrm{nA}$, in good agreement with the reverse leakage current of the experimental curves. A more detailed analysis of the forward bias nonlinearities would require measurements as function of temperature for an independent derivation of the barrier height and would introduce parameters representing the internal physical characteristics of the junctions as suggested by Tung [6].

\section{B. Magnetic Properties}

Control of the roughness of the metallic films was achieved by varying the electrodeposition potential. Nonpatterned Si samples were used for the electrodeposition of Ni films using constant electrochemical potentials from -1.1 to $-1.8 \mathrm{~V}$, which

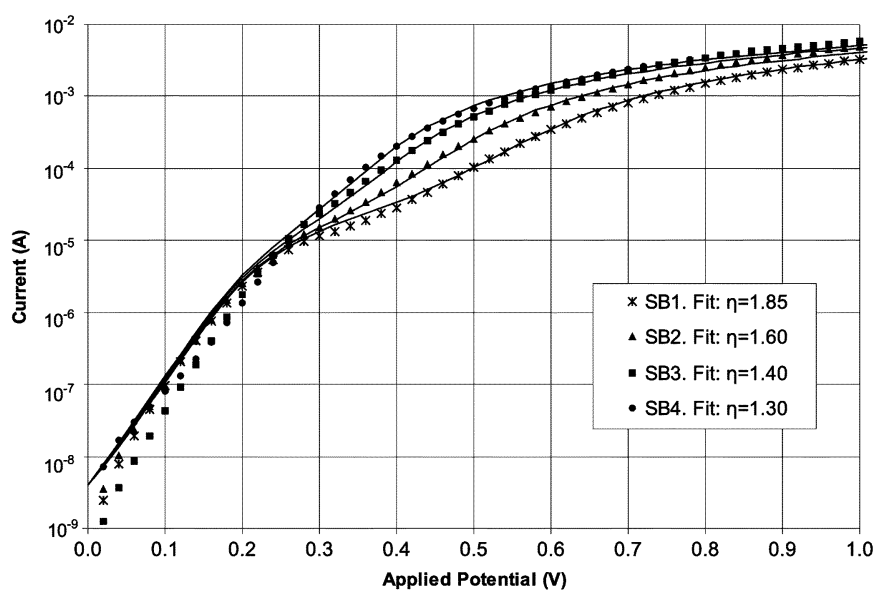

Fig. 2. Fitting of four nominal identical Ni-Si $(1-2 \Omega \cdot \mathrm{cm}) \mathrm{SBs}$ prepared on a single substrate with the phenomenological model for inhomogeneous SBs as discussed in the text. The contacts are square with side $0.07 \mathrm{~cm} . \mathrm{R}_{S 1}=6 \mathrm{k} \Omega$, $\mathrm{R}_{S 2}=100 \Omega, \mathrm{I}_{S 1}=1.9 \mathrm{nA}, \mathrm{I}_{S 2}=2 \mathrm{nA}$.

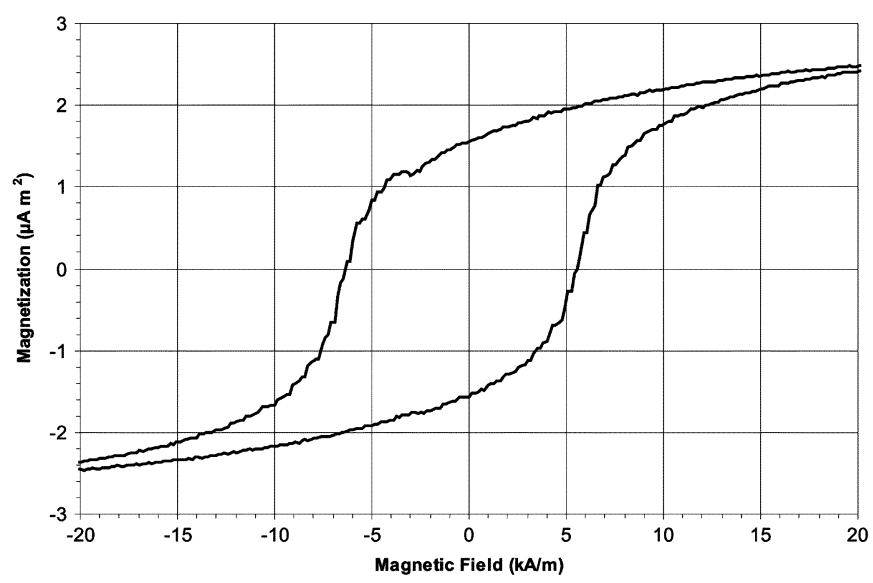

Fig. 3. Hysteresis loop of a 300-nm-thick Ni film electrodeposited on Si.

lead to different electrodeposition rates. The surface of the resulting films was characterized using AFM.

The smoothest surfaces and smallest grains were achieved for the potential of $-1.3 \mathrm{~V}$. Lower absolute potential values could not provide a uniform electrochemical nucleation, resulting in increased roughness. Higher absolute potential values lead to very fast film growth giving even rougher and intensively dendroid surfaces with high spikes.

Magnetic measurements were performed using a VSM to investigate the impact of the surface morphology on the magnetic properties of the Ni films. A typical hysteresis loop from a 300-nm-thick Ni film electrodeposited on $\mathrm{Si}$ is illustrated in Fig. 3. Table I shows correlation among the electrodeposition potential, the root-mean-square (RMS) roughness, and the coercivity values.

The coercivity increases with the surface roughness, in good agreement with other reports on similar magnetic films [4]. This effect can be attributed to roughness induced domain wall pinning contributions to coercivity [7]. From magnetization measurements the film thickness was calculated to be $300 \mathrm{~nm}$. The charge corresponding to this Ni mass revealed that $10 \%$ of the charge reaching the $\mathrm{Si}$ substrate during electrodeposition was 
TABLE I

SuRfaCE AND MAGNETIC PROPERTIES CORRELATION

\begin{tabular}{ccc}
\hline $\begin{array}{c}\text { Electrodeposition Potential } \\
(\mathrm{V})\end{array}$ & $\begin{array}{c}\text { RMS Roughness } \\
(\mathrm{nm})\end{array}$ & $\begin{array}{c}\text { Coercivity } \\
(\mathrm{Oe})\end{array}$ \\
\hline-1.1 & 17.9 & 74.9 \\
-1.3 & 15.2 & 70.8 \\
-1.4 & 19.1 & 76.9 \\
-1.6 & 237 & 80.1 \\
-1.8 & 608 & 87.2 \\
\hline \hline
\end{tabular}

Different electrodeposition potentials result in films with different roughnesses. The RMS roughness is correlated with the magnetic coercivity.
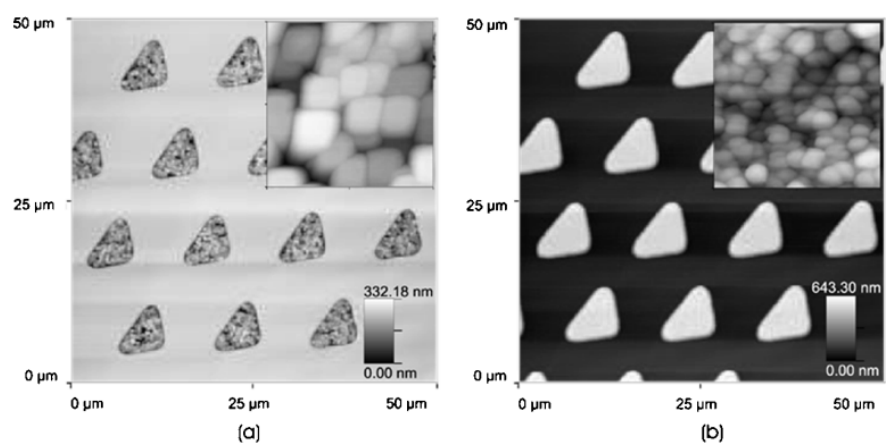

Fig. 4. AFM images of Ni electrodeposited on patterned Si showing roughness reduction by using electrochemical pulse pretreatment: (a) $1.5 \mathrm{~V}$ for $0.2 \mathrm{~s}$, (b) $1.7 \mathrm{~V}$ for $0.4 \mathrm{~s}$. In the insets, AFM scans of $2 \mu \mathrm{m} \times 2 \mu \mathrm{m}$ are presented.

due to hydrogen evolution. These considerations allow the control of the Ni film's thicknesses.

Further reduction of the surface roughness is achieved by applying pulsed electrochemical pretreatment of the Si surface before the film deposition stage. As the electrochemical nucleation rate is proportional to the applied current density, a high, short duration pulse just before electrodeposition leads to smoother Ni layers. AFM images of two Ni films electrodeposited on patterned Si using pulsed pretreatment and cyclic voltametry are shown in Fig. 4. A pulse of $-1.5 \mathrm{~V}$ for $0.2 \mathrm{~s}$ gave films with $37.1 \mathrm{~nm}$ rms roughness whereas a pulse of $-1.7 \mathrm{~V}$ for $0.4 \mathrm{~s}$ gave a much smoother film of $7.1 \mathrm{~nm}$ rms roughness. Further reduction of the film roughness is necessary for spintronic applications.

\section{Electrodeposition on Resistive Si}

A back-contact is not always practical in integrated circuits, and hence we also investigated the direct electrodeposition of $\mathrm{Ni}$ on $\mathrm{Si}$ without metallic contacts. Cyclic voltametry measurements show that it is possible to do electrodeposition even on $\mathrm{Si}$ of high resistivities. The only visible effect of high resistivity is the ohmic voltage drop in the substrate which can be modeled accurately using Poisson's equation as shown in Fig. 5.

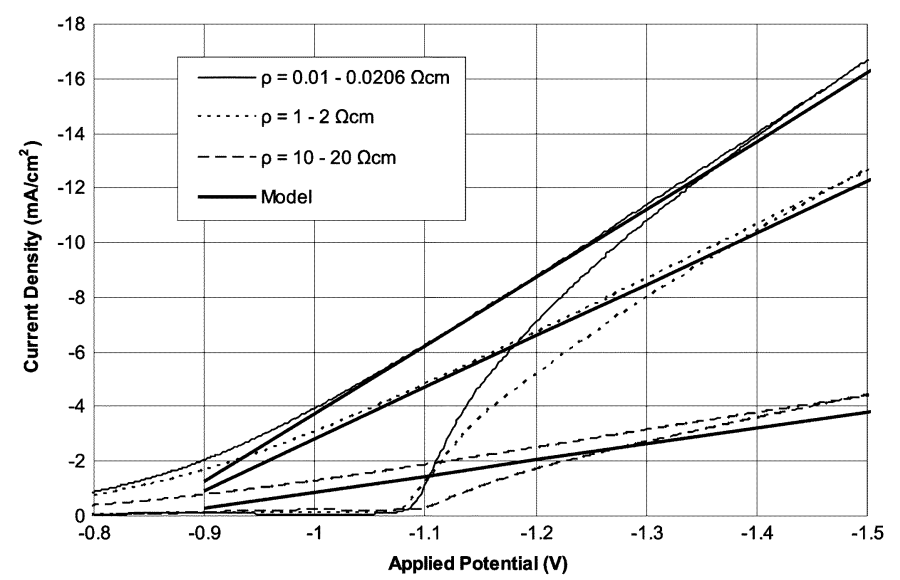

Fig. 5. Cyclic voltametry curves of Ni electrodeposition on Si with different resistivities. The thick solid lines correspond to a Poisson model with the $\mathrm{Si}$ resistivity as the only varying parameter.

\section{CONCLUSION}

The Schottky barrier of Ni-Si junctions fabricated by electrodeposition was characterized. It was shown that high quality barriers are obtained using Si with resistivity $1-2 \Omega \cdot \mathrm{cm}$, with very low reversed leakage. The roughness of the magnetic films is correlated to their coercivity. This study opens up the way for the investigation of spintronics effects on Si and consequently for the exploitation of all the benefits of Si technology.

\section{ACKNOWLEDGMENT}

This work was supported in part by the EPSRC under Grants GR/S13200/01 and GR/S49162/01.

\section{REFERENCES}

[1] D. J. Monsma, J. C. Lodder, T. J. A. Popma, and B. Dieny, "Perpendicular hot electron spin-valve effect in a new magnetic field sensor: The spin-valve transistor.," Phys. Rev. Lett., vol. 74, no. 26, pp. 5260-5263, 1995.

[2] X. J. S. van Dijken and S. S. P. Parkin, "Comparison of magnetocurrent and transfer ratio in magnetic tunnel transistors with spin-valve bases containing $\mathrm{Cu}$ and $\mathrm{Au}$ spacer layers.," Appl. Phys. Lett., vol. 82, no. 5, pp. 775-777, 2003.

[3] P. N. Bartlett, M. A. Ghanem, I. S. E. Hallag, P. de Groot, and A. Zhukov, "Electrochemical deposition of macroporous magnetic networks using colloidal templates.," J. Mater. Chem., vol. 13, pp. 2596-2602, 2003.

[4] M. L. Munford, M. L. Sartorelli, L. Seligman, and A. A. Pasa, "Morphology and magnetic properties of Co thin films electrodeposited on Si.," J. Electrochem. Soc., vol. 149, pp. C274-C279, 2002.

[5] Q. T. Zhao, U. Breuer, E. Rije, S. Lenk, and S. Mantl, "Tuning of NiSi/Si Schottky barrier heights by sulfur segregation during Ni silicidation.,' Appl. Phys. Lett., vol. 86, no. 062 108, 2005.

[6] R. T. Tung, "Electron transport at metal-semiconductor interfaces: General theory.," Phys. Rev. B, vol. 45, no. 23, pp. 13 509-13 523, 1992.

[7] S. A. Doherty, J. G. Zhu, M. Dugas, S. Anderson, and J. Tersteeg, "Correlation between evolving magnetic and morphological properties in magnetic multilayers.," IEEE Trans. Magn., vol. 34, no. 4, pp. 840-842, Jul. 1998.

Manuscript received February 4, 2005. 